\title{
All-Optical NRZ-DPSK to RZ-OOK Format Conversion Using Optical Delay Line Interferometer and Semiconductor Optical Amplifier
}

\author{
Emma Lazzeri, ${ }^{1, *}$ An Truong Nguyen, ${ }^{1}$ Giovanni Serafino, ${ }^{1}$ Nobuyuki Kataoka, ${ }^{2}$ Naoya Wada, ${ }^{2}$ Luca Ascari, ${ }^{1}$ \\ Antonella Bogoni, ${ }^{3}$ and Luca Poti ${ }^{3}$ \\ ${ }^{1}$ Scuola Superiore Sant'Anna, Via G. Moruzzi, 1, 56124 Pisa, Italy \\ ${ }^{2}$ National Institute of Information and Communications Technology, Koganei, Tokyo, 184-8795 Japan \\ ${ }^{3}$ Consorzio Nazionale Interuniversitario per le Telecomunicazioni, Via G. Moruzzi, 1, 56124 Pisa, Italy \\ "Corresponding author: emma.lazzeri@cnit.it
}

\begin{abstract}
We describe an all-optical NRZ-DPSK to RZ-OOK converter employing an optical delay line interferometer and a semiconductor optical amplifier. System penalty at $10 \mathrm{Gbit} / \mathrm{s}$ is experimentally demonstrated to be less than $1 \mathrm{~dB}$ at BER of $10^{-9}$.

(C)2010 Optical Society of America

OCIS codes: (200.4740) Optical processing, (060.4080) Modulation, (060.4510) Optical communications, (170.4090)

Modulation techniques.
\end{abstract}

\section{Introduction}

Next generation communication networks are a hybrid scenario where different technologies come to play due to the large variety of applications and to their hierarchal structure that implies diverse specifications for the networks segments (backbone, metro and access). In this picture, an adequate modulation format that meets the needs of a specific network segment is mandatory; therefore, network elements able to serve as modulation format converters are required. Nowadays, various types of modulation formats are used in optical networks, and new kinds of format are extensively studied and validated [1]. Information is carried by bits shaped into return-to-zero (RZ) or nonreturn-to-zero (NRZ) pulses: the former is suitable for optical time division multiplexing (OTDM) system due to its short duration while the latter is preferable for wavelength division multiplexing (WDM) system thanks to its narrow bandwidth. In on-off keying (OOK) modulation, the information is transferred into the optical intensity of the signal, whereas the phase of a pulse carries the information in phase-shift keying (PSK) modulation. Commonly used differential-phase-shift keying (DPSK) modulation codes the phase change of two adjacent bits to avoid the generation of an absolute phase difference. The state of the art of optical networks reveals that DPSK modulation is more suitable in high speed long haul transmission, while RZ-OOK is the best solution in short reach network segments [2,3]. The use of RZ and NRZ pulses and different kind of modulation formats in emerging networks requires an all-optical gateway element able to convert signals to make the information run across different segments transparently as well as overcome the electronics limits in terms of bit rate and bandwidth.

Considerable efforts have been spent during the last years to develop format conversion schemes. In [4] a 10Gbit/s NRZ to RZ data format conversion is achieved by means of a semiconductor optical amplifier (SOA), while in [5] a scheme based on periodically poled lithium niobate loop mirror was designed to perform carrier suppressed RZ to RZ conversion at 40GHz. Format conversions from NRZ to BPSK and QPSK was obtain by means of silicon microring resonators [6], and parallel SOA-MZI [7]. In [8], a NRZ to RZ modulator based on optical delay interferometer that could serve as format converter is presented; however this scheme is not suitable to convert NRZ signals to RZ-OOK because it generates a RZ signal where the phase is pattern dependent and related to the input NRZ properties, i.e. extinction ratio and noise.

In this paper, the first scheme to achieve NRZ-DPSK to RZ-OOK conversion based on split and delay interferometer and SOA is presented and experimentally evaluated by bit error rate (BER) measurements that show error free operation for the converted signal. The scheme also converts the signal's wavelength and exhibits timing and amplitude signal regeneration due to the use of a local optical clock signal.

\section{Experimental setup}

Fig. 1 shows the experimental setup for the NRZ-DPSK to RZ-OOK converter, the DPSK spectrum, and the eye diagrams of the converted signals taken by an Agilent sampling oscilloscope with a bandwidth of $700 \mathrm{GHz}$. The NRZ-DPSK signal generator includes a continuous wave (CW) laser source at $1550 \mathrm{~nm}$ and a phase modulator (PM) driven by a $10 \mathrm{GHz}$ pseudo random binary sequence (PRBS) $2^{15}-1$ from the bit pattern generator (BPG). NRZ-DPSK data stream is created thanks to the properties of the PRPS which is an exact DPSK version of itself after delaying a 
certain number of bits. A passive mode locking laser (MLL) driven at the same repetition rate generates a RZ optical clock at 1560nm with 3ps full-width-at-half-maximum (FWHM). Such a short pulse is chosen to allow the converted output being multiplexed into an OTDM stream working at much higher bit rate (up to 160Gbit/s) as the converter is supposed to be in a gateway between WDM and OTDM systems. A split and delay interferometer acts as a signal decoder and decodes the NRZ-DPSK into an OOK. The interferometer has two output arms: on one arm the delayed signals are summed, on the other they are subtracted; here only the arm corresponding to the subtracted signals is used and therefore the decoded data appears in its inverted version. The resulting signal is then amplified by means of an erbium doped fiber amplifier (EDFA), filtered with a $1 \mathrm{~nm}$ band pass filter (BPF), and sent to the NRZ to RZ converter based on a single semiconductor optical amplifier. The SOA (CIP SOA-XN-OEC-1550) is used as a nonlinear medium and both cross gain modulation (XGM) and polarization rotation (XPR) effects are exploited [9]. The RZ clock at 1560nm acting as probe signal is fed into the SOA, and the pump signal, coming from the NRZ-DPSK to NRZ-OOK converter, is used to saturate the amplifier gain through carrier depletion causing XGM. The gain saturation induced by the pump signal when its logic state is equal to 1 considerably reduces the probe signal power at the SOA output. On the contrary, when the pump is not present, the probe is amplified and its logic state is transferred to the SOA output. This way, the modulation is inverted again to complement the first time caused by the DPSK decoder, and the correct data are transferred to the RZ probe. To improve the extinction ratio of the converted signal, the BPF at the SOA output was chosen to have a bandwidth narrower than the probe signal one and it was slightly detuned from the signal original central wavelength of $1560 \mathrm{~nm}$. It is well known that selecting the blue sideband of the spectrum of the probe light it is possible to utilize fast chirp dynamics introduced by the amplifier, and to overcome the slow gain recovery [10].

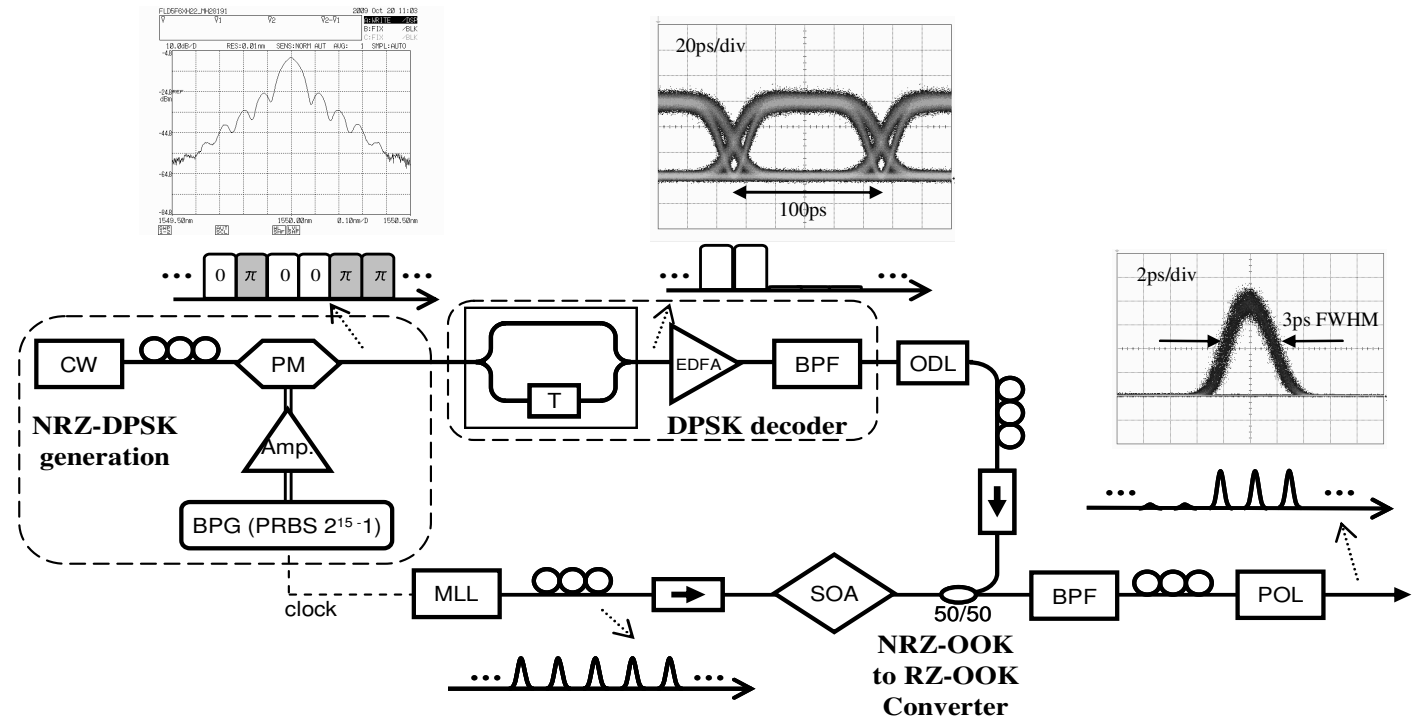

Fig. 1. Experimental Setup for NRZ-DPSK to RZ-OOK conversion

The high power NRZ-OOK signal also influences the amplifier birefringence which is the physical principle responsible for the XPR effect [11]. Depending on the NRZ-OOK signal intensity, XPR is used to differentiate the output logic states and clear the residual zeros due to the incomplete gain suppression by using a polarization controller (PC) and a polarizer at the SOA output.

The use of an uncorrupted optical clock signal as probe for the SOA makes it possible to transfer the information on a clear signal, thus regenerating the data both in amplitude and in timing.

\section{Results and discussions}

To provide a complete characterization of the format converter, BER measurements were performed on the output and on the back to back NRZ-DPSK signals by means of the receiver shown in fig. 2a, where the signal under test is amplified by two 40mW EDFAs driven at the maximum current and filtered by two 5nm BPFs. The mean power at the input of the first EDFA was varied keeping constant the average power at the receiver input to measure the bit error rate. While performing back to back measurement, a DPSK decoder was used together with the balanced detector, while only one arm of the receiver was used for evaluating the output signal performance. A minor loss of 
0.3 dB was measured for the DPSK decoder and a 3dB gain for the NRZ-DPSK BER has to be considered due to the balanced detection.

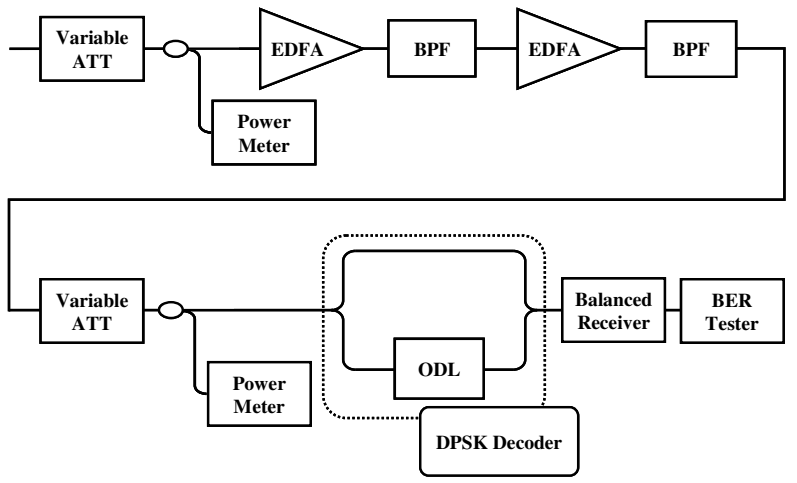

a)

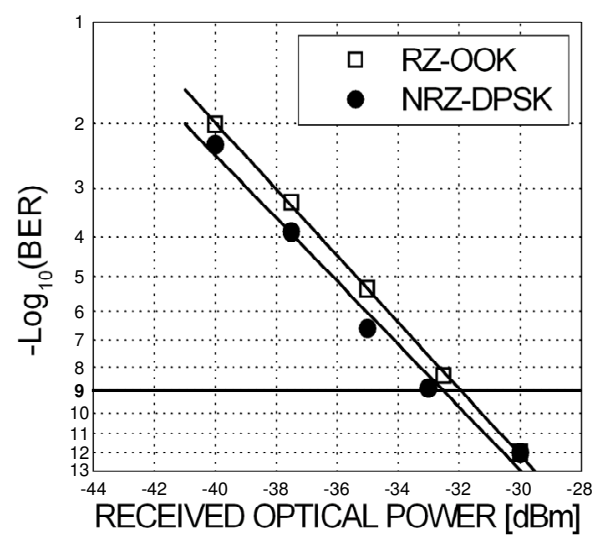

b)

Fig. 2. a) BER measurement setup, b) BER results.

Measurements results are shown in fig. 2b. Back to back NRZ-DPSK and converted RZ-OOK data streams BER are depicted in open square and solid dots respectively. On the graph, solid superimposed lines show tendencies for the two curves. Error free operations were achieved for both the signal under test and less than $1 \mathrm{~dB}$ power penalty was measured at a BER of $10^{-9}$, which is acceptable for its purpose.

\section{Conclusions}

We have demonstrated an all-optical NRZ-DPSK to RZ-OOK converter based on split and delay interferometer and XGM and XPR in SOA together with its BER measurements using a 10Gb/s PRBS $2^{15}-1$. Error-free operations are obtained for the converted signal and a comparison with the original data NRZ-DPSK data stream by means of the same receiver shows minor power penalty. The proposed converter could find its natural applications in gateway node connecting optical network segments exploiting different kind of modulation formats and multiplexing techniques.

\section{Acknowledgements}

This work was supported in part by the Italian Ministry of Foreign Affairs through the bilateral project SCOOPS. The authors would like to thank H. Sumimoto, T. Makino and T. Hashimoto from NICT for their technical support.

\section{References}

[1] G. Charlet, "Progress in optical modulation formats for high-bit rate WDM transmissions," IEEE J. Selected Topics in Quantum Electron., $12,469-483$ (2006).

[2] T. Mizuochi, K. Ishida, T. Kobayashi, J. Abe, K. Kinjo, K. Motoshima, and K. Kasahara, "A comparative study of DPSK and OOK WDM transmission over transoceanic distances and their performance degradations due to nonlinear phase noise," J. Lightwave Technol., 21, 1933-1943 (2003).

[3] A. H. Gnauck, G. Raybon, S. Chandrasekhar, J. Leuthold, C. Doerr, L. Stulz, and E. Burrows, "25×40-Gb/s copolarized DPSK transmission over 12×100-km NZDF with 50-GHz channel spacing," IEEE Photon. Technol. Lett., 15, 467-469 (2003).

[4] Gong-Ru Lin, Kun-Chieh Yu, and Yung-Cheng Chang, "10 Gbit/s all-optical non-return to zero-return-tozero data format conversion based on a backward dark-optical-comb injected semiconductor optical amplifier", Opt. Lett., 31, 10, 1376-1378 (2006).

[5] Jian Wang, Junqiang Sun, Xinliang Zhang, Dexiu Huang, and M. M. Fejer, "Optical phase erasure and its application to format conversion through cascaded second-order processes in periodically poled lithium niobate", Opt. Lett., 33, 16, 1804 -1806 (2008).

[6] Yuanyuan Lu, Fangfei Liu, Min Qiu, and Yikai Su, "All-optical format conversions from NRZ to BPSK and QPSK based on nonlinear responses in silicon microring resonators", Opt. Expr., 15, 21, 14275- 14282 (2007).

[7] Ken Mishina, Suresh M. Nissanka, Akihiro Maruta, Shunsuke Mitani, Kazuyuki Ishida, Katsuhiro Shimizu, Tatsuo Hatta, and Ken-ichi Kitayama, "All-optical modulation format conversion from NRZ-OOK to RZ-QPSK using parallel SOA-MZI OOK/BPSK converters", Opt. Expr., 15, 12, 7774-7785 (2007).

[8] P.J. Winzer and J. Leuthold, "Return-to-zero modulator using a single NRZ drive signal and an optical delay interferometer," IEEE Photon. Technol. Lett., 13, 12, 1298 - 1300, (2001).

[9] Scaffardi M., Ghelfi P., Lazzeri E., Potì L., and Bogoni A. "Photonic processing for digital comparison and full addition based on semiconductor optical amplifiers", IEEE J. of Select. Topics in Quantum Electron., 14, 3, 826-833 (2008).

[10] Y. Liu, E. Tangdiongga, Z. Li, S. Zhang, H. de Waardt, G. D. Khoe, and H. J. S. Dorren, "Error-free all-optical wavelength conversion at $160 \mathrm{~Gb} / \mathrm{s}$ using a semiconductor optical amplifier and an optical band pass filter," J. Lightw. Technol., 24, 1, 230-236, (2006).

[11] H. Soto, D. Erasme, and G. Guekos, "Cross-polarization modulation in semiconductor optical amplifiers," IEEE Photon. Technol. Lett., 11, 8, 970-972 (1999). 